\title{
Models of Hot Cores with Complex Molecules
}

\section{Susanna L. Widicus Weaver ${ }^{1}$, Robin T. Garrod ${ }^{2}$, Jacob C. Laas ${ }^{1}$, and Eric Herbst ${ }^{3}$}

\author{
${ }^{1}$ Department of Chemistry, Emory University \\ 1515 Dickey Drive, Atlanta, GA, 30322, USA \\ email: susanna.widicus.weaver@emory.edu \\ ${ }^{2}$ Department of Astronomy, Cornell University \\ 304 Space Sciences Building, Ithaca, NY, 14853, USA \\ email: rgarrod@astro.cornell.edu \\ ${ }^{3}$ Departments of Physics, Chemistry and Astronomy, The Ohio State University \\ Physics Research Building, 191 West Woodruff Avenue, Columbus, OH, 43210 \\ email: herbst.6@osu.edu
}

\begin{abstract}
Recent models of hot cores have incorporated previously-uninvestigated chemical pathways that lead to the formation of complex organic molecules (COMs; i.e. species containing six or more atoms). In addition to the gas-phase ion-molecule reactions long thought to dominate the organic chemistry in these regions, these models now include photodissociation-driven grain surface reaction pathways that can also lead to COMs. Here, simple grain surface ice species photodissociate to form small radicals such as $\mathrm{OH}, \mathrm{CH}_{3}, \mathrm{CH}_{2} \mathrm{OH}, \mathrm{CH}_{3} \mathrm{O}, \mathrm{HCO}$, and $\mathrm{NH}_{2}$. These species become mobile at temperatures above $30 \mathrm{~K}$ during the warm-up phase of star formation. Radical-radical addition reactions on grain surfaces can then form an array of COMs that are ejected into the gas phase at higher temperatures. Photodissociation experiments on pure and mixed ices also show that these complex molecules can indeed form from simple species. The molecules predicted to form from this type of chemistry reasonably match the organic inventory observed in high mass hot cores such as Sgr B2(N) and Orion-KL. However, the relative abundances of the observed molecules differ from the predicted values, and also differ between sources. Given this disparity, it remains unclear whether grain surface chemistry governed by photodissociation is the dominant mechanism for the formation of COMs, or whether other unexplored gas-phase reaction pathways could also contribute significantly to their formation. The influence that the physical conditions of the source have on the chemical inventory also remains unclear. Here we overview the chemical pathways for COM formation in hot cores. We also present new modeling results that begin to narrow down the possible routes for production of $\mathrm{COMs}$ based on the observed relative abundances of methyl formate $\left(\mathrm{HCOOCH}_{3}\right)$ and its $\mathrm{C}_{2} \mathrm{H}_{4} \mathrm{O}_{2}$ structural isomers.
\end{abstract}

Keywords. astrochemistry, molecular processes, methods: numerical, stars: formation, ISM: abundances, ISM: clouds, ISM: molecules

\section{Introduction}

Over 160 molecules have now been detected in interstellar and circumstellar environments, and a large fraction of these molecules are classified as complex organic molecules, or COMs (i.e. those organic species containing six or more atoms; Herbst \& van Dishoeck 2009). The majority of COMs have been discovered in the warm environments of hot cores, where it was presumed that gas-phase ion-molecule reactions were the predominant formation mechanism (Charnley 2001). However, theoretical and experimental investigations of the possible gas-phase formation pathways for the highly-abundant COM 
methyl formate $\left(\mathrm{HCOOCH}_{3}\right)$ showed that its formation through these types of reactions cannot explain its observed abundance (Horn et al. 2004). In addition, methyl formate has been detected in regions where the timescales are too short for sufficient build-up of COMs through gas-phase reactions. Such regions include shocked regions in the Galactic Center (Requena-Torres et al. 2006), low-mass hot cores (Ceccarelli 2005), and cold envelopes around embedded protostars (Öberg et al. 2010). These discoveries call into question the gas-phase formation mechanisms previously proposed for methyl formate and other related COMs.

Recent astrochemical modeling studies (Garrod \& Herbst 2006; Garrod et al. 2008) have invoked grain surface radical-radical reactions to explain the high abundance of methyl formate in hot cores. Additional studies (Laas et al. 2011) have further investigated the relative abundance of methyl formate to its $\mathrm{C}_{2} \mathrm{H}_{4} \mathrm{O}_{2}$ isomers acetic acid $\left(\mathrm{CH}_{3} \mathrm{COOH}\right)$ and glycolaldehyde $\left(\mathrm{HOCH}_{2} \mathrm{CHO}\right)$. An overview of these hot core chemical models and associated results is presented here.

\section{Chemical Models of Hot Cores}

\subsection{Previous Hot Core Models}

Previous models of hot cores assumed limits on interstellar chemical reactions based on the characteristic temperatures of interstellar clouds. The assumption was long made that only simple single-atom addition reactions could occur on grain surfaces, and that the more complex chemistry occurred through gas-phase ion-molecule reactions in warmer regions of star formation. Indeed, at $10 \mathrm{~K}$, only single atoms are mobile on an ice surface, limiting the possible condensed-phase chemistry to the formation of simple molecules such as water and methanol. The physical model assumed in these previous studies for the evolution of the hot core was a single temperature jump, where the simpler molecules trapped in ices in cold clouds are injected into the gas phase through thermal evaporation from the grains. A complex network of gas-phase ion-molecule chemistry was then invoked to explain the formation of COMs in hot cores. See the review of Charnley (2001) and references therein for a more comprehensive overview of these earlier hot core chemical models.

\subsection{New Gas/Grain Hot Core Models}

\subsubsection{Grain Surface Chemistry During the Warm-up Phase of Star Formation}

In the studies of Garrod \& Herbst (2006) and Garrod et al. (2008), a second-order power law was used to model the warm-up phase of a hot core. Such an approach had been suggested by Viti \& Williams (1999) to address the time-dependent evaporation of the ice mantles. This change in physical model allowed the employment of a more complex network of chemical reactions on grain surfaces at temperatures that fell within the range between the two extremes of a dense cloud $(10 \mathrm{~K})$ and a hot core $(200 \mathrm{~K})$. As in the case of the previous models, the single-atom addition reactions on grain surfaces are the most efficient reactions at colder temperatures. However, molecular species become mobile on ice surfaces above $\sim 30 \mathrm{~K}$, enabling an extensive chemical reaction network to be employed for grain surface chemistry.

The assumption is made in the model of Garrod et al. (2008) that the simple molecules formed in colder regions dissociate in the ice matrix through either direct or cosmic-ray induced photodissociation to form $\mathrm{OH}, \mathrm{CH}_{3}, \mathrm{CH}_{3} \mathrm{O}, \mathrm{CH}_{2} \mathrm{OH}, \mathrm{HCO}$, and $\mathrm{NH}_{2}$ radicals through the following processes: 


$$
\begin{aligned}
\mathrm{H}_{2} \mathrm{O} & \rightarrow \mathrm{H}+\mathrm{OH} \\
\mathrm{CH}_{3} \mathrm{OH} & \rightarrow \mathrm{CH}_{3}+\mathrm{OH} \\
& \rightarrow \mathrm{CH}_{2} \mathrm{OH}+\mathrm{H} \\
& \rightarrow \mathrm{CH}_{3} \mathrm{O}+\mathrm{H} \\
\mathrm{H}_{2} \mathrm{CO} & \rightarrow \mathrm{H}+\mathrm{HCO} \\
\mathrm{NH}_{3} & \rightarrow \mathrm{NH}_{2}+\mathrm{H}
\end{aligned}
$$

The radicals formed from this photodissociation become mobile at temperatures $>30 \mathrm{~K}$. More complex organics such as methyl formate and its structural isomers are formed through the combination reactions of these mobile radicals during the warm-up phase of star formation. The grain surface reactions leading to methyl formate and glycolaldehyde are:

$$
\begin{gathered}
\mathrm{CH}_{3} \mathrm{O}+\mathrm{HCO} \rightarrow \mathrm{HCOOCH}_{3} \\
\mathrm{CH}_{2} \mathrm{OH}+\mathrm{HCO} \rightarrow \mathrm{HOCH}_{2} \mathrm{CHO}
\end{gathered}
$$

The grain surface chemistry that leads to acetic acid is more complicated, and a sequence of three reactions is required to explain its formation:

$$
\begin{aligned}
\mathrm{CH}_{3}+\mathrm{HCO} & \rightarrow \mathrm{CH}_{3} \mathrm{CHO} \\
\mathrm{CH}_{3} \mathrm{CHO}+\mathrm{H} & \rightarrow \mathrm{H}_{2}+\mathrm{CH}_{3} \mathrm{CO} \\
\mathrm{CH}_{3} \mathrm{CO}+\mathrm{OH} & \rightarrow \mathrm{CH}_{3} \mathrm{COOH}
\end{aligned}
$$

This type of grain surface chemistry can build up significant chemical complexity in the envelopes around young stars. The molecules formed from this grain surface chemistry then evaporate at warmer temperatures. The molecules released from the ices undergo subsequent ion-molecule gas-phase reactions to form even more complex organic molecules.

The results of the Garrod et al. (2008) gas/grain model are shown in Figure 1. Here, the peak abundances predicted by the model are compared to the observed abundances in the Sagittarius B2(N-LMH) source. It is clear that the addition of radical-radical grain surface reactions has improved the agreement between the models and observations considerably, as significant abundances are predicted for a variety of COMs that have been detected in the Sgr B2(N-LMH) hot core. Despite this improvement, the relative abundances of many COMs, and in particular methyl formate and its structural isomers, are not consistent with the Sgr observations. Some results of this study made it clear that additional studies examining COM formation in these sources are required. More sophisticated hydrodynamics models, such as those employed by Aikawa et al. (2008), may also be needed to properly model the complex physical environment of a hot core. 


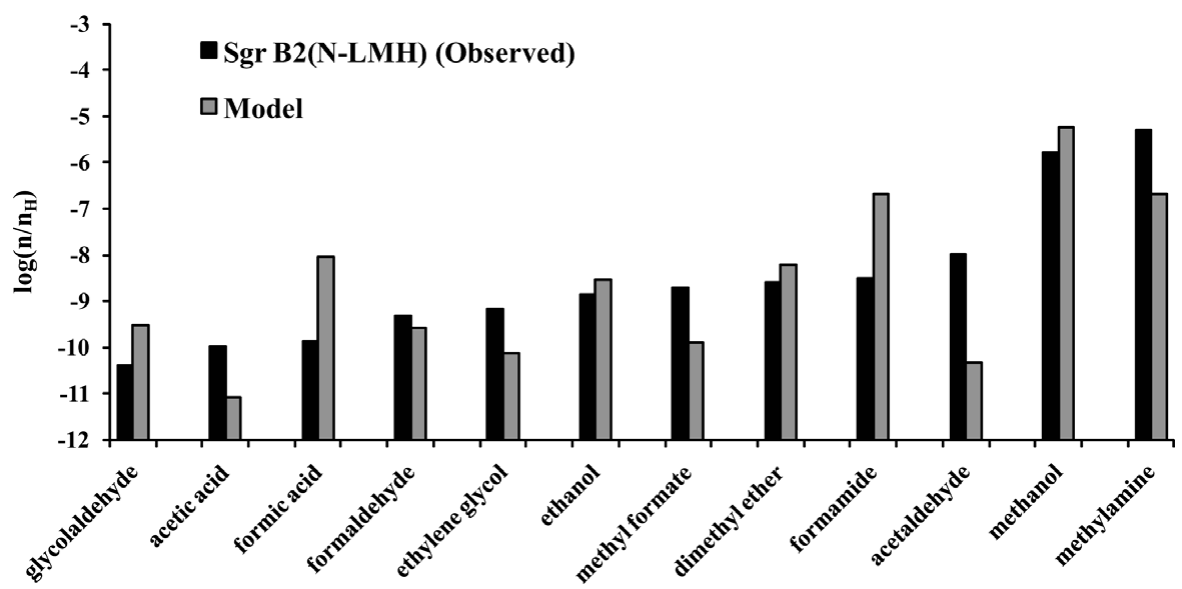

Figure 1. Results of initial gas/grain hot core model as adapted from Garrod et al. (2008). This model incorporates photodissociation-induced grain surface reactions to form complex organic molecules. Comparison is made between predicted peak abundance for each molecule in the model and the observational results for the Sgr B2(N-LMH) hot core.

\subsubsection{Testing the Influence of Methanol Photodissociation Branching Ratios}

Examination of Reactions 2.5 - 2.7 reveals that the photodissociation of methanol plays a significant role in the production of COMs through grain surface radical-radical reactions. Methanol is the most abundant organic molecule in interstellar ices and is therefore the dominant source of the $\mathrm{CH}_{3}, \mathrm{CH}_{2} \mathrm{OH}$, and $\mathrm{CH}_{3} \mathrm{O}$ radicals on grain surfaces. Methanol photodissociation is therefore the primary route to several of the major organic functional groups that make up COMs. Methanol photodissociation branching ratios therefore strongly influence structural isomerism in interstellar clouds, and are particularly crucial for methyl formate and its structural isomers.

There is little agreement in the literature as to the values for either the condensedphase or gas-phase methanol photodissociation branching ratios. A laboratory study by Öberg et al. (2009) of methanol photodissociation in interstellar ice analogs has made the first step toward determining the information required for astrochemical models. The experiment monitors the stable species that form from reactions between the photodissociation products, rather than directly monitoring the photodissociation products themselves. Therefore, the reaction rates and branching ratios derived from these ice photodissociation measurements are difficult to employ in astrochemical models because determination of branching ratios is also dependent on chemical modeling of the ice chemistry. The model employed by Öberg et al. (2009) does measure the time-dependent abundance of $\mathrm{CH}_{2} \mathrm{OH}$ and $\mathrm{H}_{2} \mathrm{CO}$, but not other methanol photolysis products.

Reports of gas-phase methanol photodissociation studies are extensive in the literature, but there is very little agreement as to the branching ratios for the dissociation pathways. This disagreement arises primarily from the methods used to monitor product formation. Hagege et al. (1968) determined the primary processes at $189.9 \mathrm{~nm}$ to be the three processes included in Reaction 2.2 above, plus the additional route

$$
\mathrm{CH}_{3} \mathrm{OH} \rightarrow \mathrm{CH}_{2} \mathrm{O}+\mathrm{H}_{2}
$$

with relative ratios of $\mathrm{CH}_{2} \mathrm{OH}+\mathrm{CH}_{3} \mathrm{O}: \mathrm{CH}_{3}: \mathrm{CH}_{2} \mathrm{O}=0.75: 0.05: 0.20$. These conclusions were based on measurements of rates of various reactions and an analysis of a kinetics 
model; these values have remained the most accepted photodissociation branching ratios to date. Thus, $\mathrm{CH}_{2} \mathrm{OH}$ and $\mathrm{CH}_{3} \mathrm{O}$ are thought to be the primary photodissociation products for methanol. A more recent gas-phase study by Satyapal et al. (1989) agrees qualitatively with the conclusions by Hagege et al. However, improved photodissociation branching ratios have not been reported in the literature because no experimental technique used for such studies distinguishes between the $\mathrm{CH}_{2} \mathrm{OH}$ and $\mathrm{CH}_{3} \mathrm{O}$ species in measurement.

The gas/grain chemical network of Garrod et al. (2008) was the first to distinguish between $\mathrm{CH}_{2} \mathrm{OH}$ and $\mathrm{CH}_{3} \mathrm{O}$, and assumed a methanol photodissociation branching ratio of $60 \%$ for the $\mathrm{CH}_{3}+\mathrm{OH}$ channel, with the remainder split between the $\mathrm{CH}_{3} \mathrm{O}$ and $\mathrm{CH}_{2} \mathrm{OH}$ channels. The recent work of Laas et al. (2011) has explored the extreme limits of these photodissociation branching ratios, with trials being conducted for each of the three limits of the methanol photodissociation mechanism. In each trial, one channel (i.e. $\mathrm{CH}_{2} \mathrm{OH}, \mathrm{CH}_{3} \mathrm{O}$, or $\mathrm{CH}_{3}$ ) was set to a branching ratio of $90 \%$, and the other two channels set to $5 \%$ each. The results of the Laas et al. (2011) study are shown in Figure 2, focusing specifically on methyl formate and its structural isomers. It was found that changes to the gas-phase photodissociation branching ratios for methanol had little effect on COM chemistry, as would be expected given that the major production routes for most COMs are grain-surface reactions. In contrast, changes to the grain surface photodissociation branching ratios for methanol significantly influence the relative abundances of COMs, particularly methyl formate and its structural isomers. As can be seen in Figure 2, the set of branching ratios favoring the $\mathrm{CH}_{3} \mathrm{O}$ channel give the best match to the Sgr B2(N-LMH) methyl formate abundance. However, the branching ratios favoring the $\mathrm{CH}_{3}$ channel give a better match to observations for acetic acid and glycolaldehyde. Additional laboratory and observational studies are required to determine the values appropriate for interstellar ices.
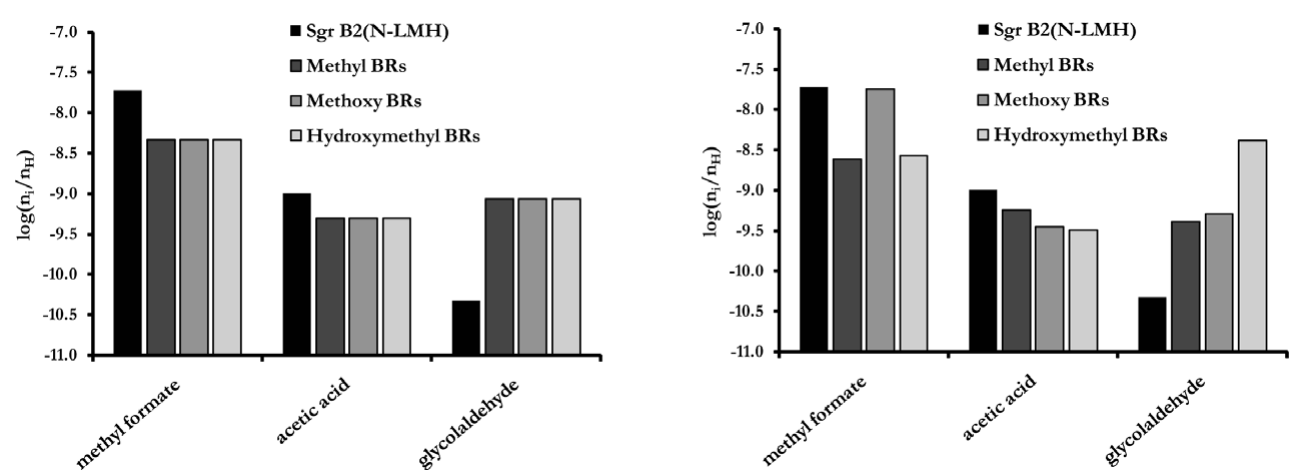

Figure 2. Peak abundances predicted by modeling studies where the methanol photodissociation branching ratios were adjusted using the gas/grain network of Garrod et al. (2008), as adapted from the results of Laas et al. (2011). The results from adjustment of the gas-phase methanol photodissociation branching ratios are shown in the left panel; the results from adjustment of the grain surface methanol photodissociation branching ratios are shown in the right panel. The trials are labeled by the name of the major photodissociation product, such that in the case of the "Methoxy BRs" trial, the $\mathrm{CH}_{3} \mathrm{O}$ branching ratio was set to 0.9 , while the $\mathrm{CH}_{2} \mathrm{OH}$ and $\mathrm{CH}_{3}$ channels were each set to a branching ratio value of 0.05 .

In addition to investigating the methanol photodissociation branching ratios, the Laas et al. (2011) study examined the influence of warm-up timescale on COM chemistry. The results of this analysis are shown in Figure 3 for three warm-up timescales. These results 
show that a longer warm-up timescale favors production of COMs through grain surface chemistry. This trend arises because the cloud spends a longer time in the temperature window that favors radical-radical grain surface chemistry. Longer warm-up timescales are required to build up significant abundances of the more complex species such as acetic acid that require multiple grain surface reactions for formation.

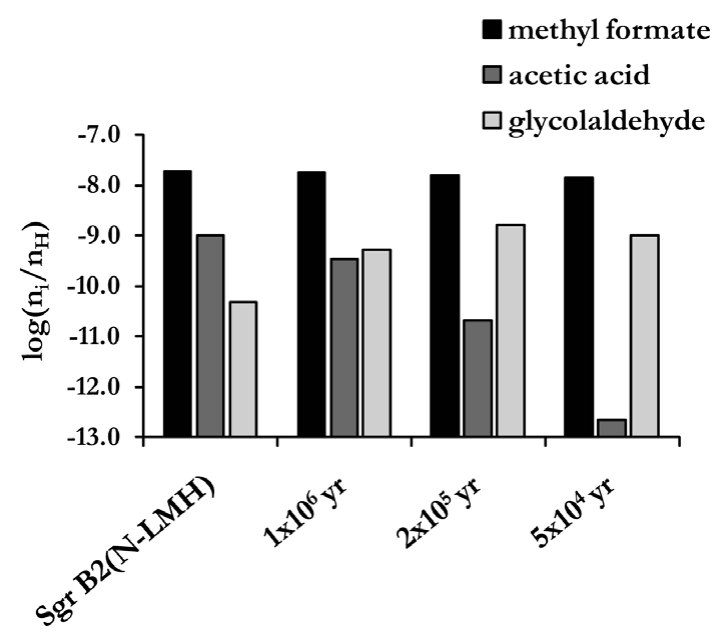

Figure 3. Results of modeling studies investigating the influence of warm-up timescale on the relative peak abundances of methyl formate and its structural isomers acetic acid and glycolaldehyde, as adapted from Laas et al. (2011).

\subsubsection{Testing Additional Gas Phase Routes to Methyl Formate}

Although the Laas et al. (2011) model gives better agreement with the observations for methyl formate over the gas/grain model of Garrod et al. (2008), it is clear that the model still needs improvement before it will match observations. New observational information has led us to investigate additional gas-phase formation pathways for methyl formate. Recent interferometric studies (Neill et al. 2011) have probed the spatial distributions of methyl formate, methanol, formic acid, and dimethyl ether in the Orion-KL star forming region. These observations show that methyl formate is spatially correlated with both methanol and dimethyl ether, but is spatially anti-correlated with formic acid. Pate and coworkers postulated that this anti-correlation might indicate a gas-phase chemical mechanism that forms methyl formate at the expense of formic acid (Neill et al. 2011). They propose two additional gas-phase formation pathways for methyl formate that have not been considered in previous astrochemical models. One such pathway is the Fischer esterification reaction between protonated formic acid and methanol to form protonated methyl formate:

$$
\mathrm{HCOOH}_{2}^{+}+\mathrm{CH}_{3} \mathrm{OH} \rightarrow \mathrm{HC}(\mathrm{OH})_{2} \mathrm{OCH}_{3}^{+}+\mathrm{H}_{2} \mathrm{O}
$$

Calculations show that this reaction has an activation energy of $\sim 3100 \mathrm{~K}$ (Neill et al. 2011), and is therefore unlikely to be an efficient formation mechanism for methyl formate under interstellar conditions. However, the methyl cation transfer reaction between protonated methanol and formic acid 


$$
\mathrm{HCOOH}+\mathrm{CH}_{3} \mathrm{OH}_{2}^{+} \rightarrow \mathrm{HC}(\mathrm{OH})_{2} \mathrm{OCH}_{3}^{+}+\mathrm{H}_{2} \mathrm{O}
$$

was found to have no activation energy when proceeding to the trans conformer of protonated methyl formate (Neill et al. 2011). The activation energy for the reaction leading to cis-protonated methyl formate was calculated to be $\sim 1320 \mathrm{~K}$ (Neill et al. 2011). The two conformers of methyl formate are structurally distinct, with the orientation of the $-\mathrm{CH}_{3} \mathrm{O}$ group relative to the $-\mathrm{CHO}$ group being the distinguishing structural difference. The cis conformer is energetically more stable, and is therefore the conformer commonly detected in hot cores. The cis conformer was calculated to be $2880 \mathrm{~K}$ lower in energy than the trans species (Neill et al. 2011). The protonated versions of the cis and trans stereoisomers have similar structures to their neutral counterparts, but in this case the trans species is lower in energy by $2880 \mathrm{~K}$ (Neill et al. 2011).

These reactions were added to the gas/grain network of Laas et al. (2011) and combined with the methoxy branching ratio trial to test their influence on the formation of methyl formate in hot cores. The results are shown in Figure 4. It was found that these reactions had no effect on the predicted peak abundance of cis-methyl formate, which is the conformer that has been detected in the interstellar medium. However, a significant amount of trans-methyl formate was predicted to form at the higher temperatures of hot cores, with fractional abundances relative to $\mathrm{H}$ approaching $10^{-11}$ at temperatures above $100 \mathrm{~K}$. Pate and coworkers have now obtained the laboratory spectrum of transmethyl formate, and report its preliminary detection in the Sgr B2(N-LMH) hot core (Neill et al. 2011), in agreement with the model prediction. It should be noted here that this detection is not in Orion-KL, where the anti-correlation between formic acid and methyl formate emission has been observed in imaging studies. Additional observations are necessary to examine the spatial distributions of these molecules in Sgr B2 and search for trans-methyl formate in Orion-KL.

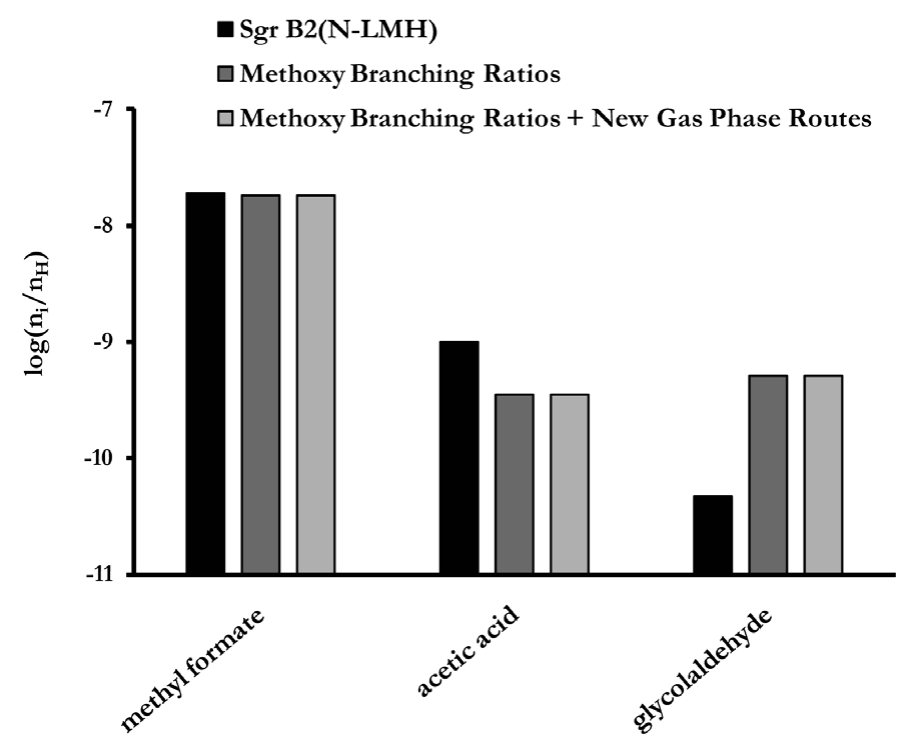

Figure 4. Results of modeling studies investigating the influence of two new gas-phase formation routes for methyl formate, as adapted from Laas et al. (2011). 
The trans conformer of methyl formate may serve as an excellent tracer of gas-phase chemistry in star-forming regions. Its predominant formation mechanism is the electron recombination reaction of protonated trans-methyl formate, which only forms from the gas-phase methyl cation transfer reaction between protonated methanol and formic acid. The assumption is made that trans-methyl formate forms exclusively through this gasphase reaction pathway in hot cores, and no grain surface chemistry contributes directly to its abundance. The basis for this assumption is that the two methyl formate conformers are not distinguished on the grain surface, and any methyl formate liberated from grains will be deposited into the gas phase with the expected thermal distribution based on the relative energies of the two conformers. It is not clear if this assumption is valid, and additional investigation of stereochemical effects should be considered in future work. However, identification of additional molecules that can serve as tracers of grain surface or gas phase chemistry would be an important step forward in understanding the chemical mechanisms at play in interstellar clouds.

\section{Conclusions}

Previous interstellar chemical models, in which gas-phase ion-molecule reactions were assumed to dominate the formation of complex molecules, could not satisfactorily explain the observed abundances of COMs in hot cores. New gas/grain models that incorporate a warm-up model for star formation show that significant abundances of COMs are predicted to form from photodissociation-induced radical-radical reactions on grain surfaces. Additional studies have investigated the formation of methyl formate and its structural isomers acetic acid and glycolaldehyde, and found that the branching ratios for methanol photodissociation on grains strongly influence the relative abundances of these COMs. The timescale for warm-up also greatly influences COM production, with longer warmup timescales favoring more complex chemistry. Future work will examine the possibility for a higher level of COM complexity being reached through grain surface reactions.

In addition to these studies involving grain surface chemistry, recent interferometric observations indicate an anti-correlation between methyl formate and formic acid in the Orion-KL region, prompting studies of additional gas-phase formation mechanisms for methyl formate that involve a formic acid precursor. These studies show that the addition of two new pathways to methyl formate through its protonated precursor do not influence the predicted peak abundance of the cis conformer of methyl formate (which is the one detected in interstellar clouds). These two reactions are the Fischer esterification reaction between protonated formic acid and methanol and the methyl cation transfer reaction between protonated methanol and formic acid. Despite the inefficient formation of cismethyl formate through these processes, the methyl cation transfer reaction does have a barrierless reaction pathway leading to the trans stereoisomer of protonated methyl formate. A large abundance of trans-methyl formate is predicted at temperatures above $100 \mathrm{~K}$ in hot cores. Follow-up laboratory and observational studies have confirmed the presence of this methyl formate conformer in Sgr B2(N-LMH). This conformer may serve as an excellent tracer of gas phase chemistry in interstellar clouds because there are no grain-surface reactions that directly form trans-methyl formate. Full quantitative understanding of the chemical mechanisms in interstellar clouds requires identification of additional molecules that can serve as tracers of grain surface or gas phase chemistry. Additional studies will examine other such barrierless chemical pathways involving known COMs. Observational studies that enable comparison to sources other than Orion-KL and Sgr B2(N) can also provide crucial information to constrain models of COM chemistry in hot cores. 


\section{References}

Aikawa, Y., Wakelam, V., Garrod, R. T., \& Herbst, E. 2008, ApJ, 674, 984

Charnley, S. 2001, in: F. Giovanelli (ed.), The Bridge Between the Big Bang \&J Biology, (Rome: Cons. Naz. d. Ricerche), p. 139

Ceccarelli, C. 2006, in: D. C. Lis, G. A. Blake, \& E. Herbst (eds.), Astrochemistry: Recent Successes and Current Challenges, Proc. IAU Symposium 231 (Cambridge, UK: Cambridge Univ. Press), p. 1

Garrod, R. T. \& Herbst, E. 2006, A\& A, 457, 927

Garrod, R. T., Widicus Weaver, S. L., \& Herbst, E. 2008, ApJ, 682, 283

Hagege, J., Roberge, P. C., \& Vermeil, C. 1968, Trans. Faraday Soc., 64, 3288

Herbst, E. \& van Dishoeck, E. F. 2009, ARAA, 47, 427

Horn, A., Møllendal, H., Sekiguchi, O., Uggerud, E., Roberts, H., Herbst, E., Viggiano, A. A., \& Fridgen, T. D. 2004, ApJ, 611, 605

Laas, J. C., Garrod, R. T., Herbst, E., \& Widicus Weaver, S. L. 2011, ApJ, 728, 71

Neill, J. L., Steber, A. L., Muckle, M. T., Zaleski, D. T., Lattanzi, V., Spezzano, S., McCarthy, M. C., Remijan, A. J., Friedel, D. N., Widicus Weaver, S. L., \& Pate, B. H. 2011, J. Phys. Chem. A, 115, 6472

Öberg, K. I., Garrod, R. T., van Dishoeck, E. F., \& Linnartz, H. 2009, A\&A A, 504, 891

Öberg, K. I., Bottinelli, S., Jørgensen, J. K., \& van Dishoeck, E. F. 2010, ApJ, 716, 825

Requena-Torres, M. A., Martín-Pintado, J., Rodríguez-Franco, A., Martín, S., RodríguezFernández, N. J., \& de Vincente, P. 2006, A\& A, 455, 971

Satyapal, S., Park, J., Bersohn, R., \& Katz, B. 1989, J. Phys. Chem., 91, 6873

Viti, S. \& William, D. A. 1999, MNRAS, 305, 755

\section{Discussion}

FIELD: What is the role of very low energy electrons in initiating chemistry in ice mantles?

Widicus WEAVER: High-energy electrons (i.e. cosmic rays) are considered in the grain surface chemistry included in these models. However, we do not consider the influence of low-energy electrons here.

GuÉLIN: Sgr B2 is a distant source and current millimeter-wave observations have a low spatial resolution and mix-up different types of sources (hot cores, cold clouds, ...). You should really compare your model results with observations of well resolved sources, such as Orion KL hot cores. There, it seems that acetic acid is hardly or not detected, whereas methyl formate is very strong (e.g. C. Favre et al. A\&A 2011).

Widicus WeAver: Sgr B2 is not the most ideal source for comparison to these models because it is clear that both the chemical and physical environment here is very complex and unusual. Acetic acid detections in other sources are limited, and glycolaldehyde has only been detected in two sources. My group is conducting observations of a variety of sources targeting COMs so that we have additional sources to which we can compare the model predictions. 